\title{
ON THE HOMOTOPY THEORY OF MONOIDS
}

\author{
CAROL M. HURWITZ
}

(Received 10 September 1987)

Communicated by R. H. Street

\begin{abstract}
In this paper, it is shown that any connected, small category can be embedded in a semi-groupoid (a category in which there is at least one isomorphism between any two elements) in such a way that the embedding includes a homotopy equivalence of classifying spaces. This immediately gives a monoid whose classifying space is of the same homotopy type as that of the small category. This construction is essentially algorithmic, and furthermore, yields a finitely presented monoid whenever the small category is finitely presented. Some of these results are generalizations of ideas of McDuff.
\end{abstract}

1980 Mathematics subject classification (Amer. Math. Soc.) (1985 Revision): 20 M 50, 55 U 35.

In 1976, D. M. Kan and W. P. Thurston [5] proved that there is a functorial construction which associates to any path-connected space a discrete group $G$ and a map $B G \rightarrow X$ that is a homology equivalence. The kernel of the surjection $G=\Pi_{1} B G \rightarrow \Pi_{1} X$ is a perfect, normal subgroup $P$, such that the Quillen ( $)^{+}$construction applied to the pair $(G, P)$ yields a space having the same weak homology type as $X$. Central to their proof is the functorial construction of a homological cone for any discrete group, that is, a natural embedding of a group into an acyclic group. In their construction, $G$ is always uncountable even when $X$ is a finite $\mathrm{CW}$-complex. They posed the following question: given a finite $\mathrm{CW}$-complex $X$, might one produce a $K(G, 1)$ that is a finite $C W$-complex homologically equivalent to $X$. In other words, is it possible to construct a group $G$ as above whose classifying space has the homotopy type of a finite CW-complex. Such a group is said to be geometrically finite. Following this paper, G. Baumslag, E. Dyer, and A. Heller [1],

(C) 1989 Australian Mathematical Society 0263-6115/89\$A2.00+0.00 
showed that the category of pointed, connected CW-complexes with homotopy classes of maps is equivalent to a suitable category of fractions of the category of pairs $(G, P)$ where $G$ is a group, $P$ a perfect, normal subgroup. In this context they used a variant of the homological cone construction to give a construction of the group, $G$, which associates a geometrically finite group to a finite CW-complex.

Although the homology type of any path-connected space can be realized as a $K(G, 1)$, clearly this is not true of the homotopy type. However, along the lines of Kan and Thurston, D. McDuff [10] showed that every path-connected space has the weak homotopy type of the classifying space of a monoid.

With McDuff's results in mind, we see that given a small category, one can construct a monoid of the same homotopy type as the given category, that is, that of its classifying space, by going through Top. We show that, given a connected, small category, one can find an effective construction entirely within the category of small categories which yields a monoid of the same homotopy type. The construction is totally algebraic and effective. Given a category presented by generators and relations, we construct a monoid of the same homotopy type also presented by generators and relations. Furthermore, for a finitely presented category, one gets a finitely presented monoid and once the selection of a spanning tree for the given category has been made, the construction is algorithmic.

\section{Preliminaries}

Given a connected, small category, we embed it in a semi-groupoid, that is, a category having at least one isomorphism between any two objects, with the same homotopy type as the given category. At each vertex (object) of the semi-groupoid is a monoid having the same homotopy type as the semigroupoid. If the semi-groupoid is presented in terms of generators and relations, we need only fix an object and select a set of isomorphisms, one between the fixed object and each of the other objects, then pull back the generators and relations via these selected morphisms to obtain a monoid presented in terms of generators and relations.

The construction of the semigroupoid from a given category, with object set $I$, involves only categories with the same object set, thus it is convenient to work in Cat ${ }_{I}$, the subcategory of small categories all having the same object set, $I$. We begin by showing that if the initial legs of the following push-out diagram in Cat I $_{I}$ satisfy a certain freeness condition, we can generalize to small categories the word theorem in group theory for free products with amalgamation [6] and the generalization proved by McDuff for monoids 
[10]. Since groups and monoids can be thought of as small categories with one object, their elements being morphisms from that object to itself, these theorems can be viewed as special cases of a more general theorem about categories. In the presence of right-freeness, we apply the classifying functor, $B$ [12], to the push-out and use the word theorem to show that the induced map $B N^{1} \cup_{B L} B N^{2} \rightarrow B\left(N^{1} *_{L} N^{2}\right)$ is a homotopy equivalence. This is a generalization of McDuff's theorem for monoids [10] and J. H. C. Whitehead's theorem on asphericity [13].

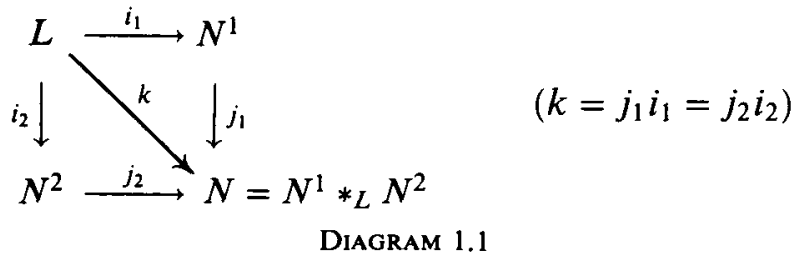

These results provide the tools necessary to construct the desired semigroupoid. This is done by constructing a mapping cylinder for an arbitrary morphism $F: L \rightarrow N$ in Cat $_{I}$, then using the mapping cylinder to embed the category in a semi-groupoid.

The above generalizations require that the arguments be stated in the language of categories. For instance, the tensor product used in an argument by McDuff [10] generalizes to the left Kan extension ([4] and [9, pages 232238]). The methods used to calculate the homology of a category are described in Hilton and Stammbach [3, pages 321-330]. We also note that if for two functors, $f$ and $g$, there exists a natural transformation $T: f \rightarrow g$, then $B T: B f \rightarrow B g$ is a homotopy and we refer to $f$ and $g$ as homotopic functors.

\section{A word theorem for push-outs in $\mathrm{Cat}_{I}$}

The notion of "right-free" inclusion we introduce below ensures that for two small categories in Cat ${ }_{l}$, one included in the other, the subcategory induces a decomposition of the larger category into disjoint "cosets" in a manner analogous to that for groups. When this definition is satisfied, each "coset" is in bijective correspondence with any other, and there is a fixed set of "coset" representatives such that any element of the larger category can be written in a unique way as the composition of a "coset" representative with an element from the smaller category. In general, the inclusion of a submonoid into a monoid is not a right-free inclusion. In the case where the categories are groups, the definition of right-free inclusion reduces to the 
statement that one group is a subgroup of another. The definition of rightfreeness generalizes from groups and monoids [10] what is needed for a word theorem in Cat . $_{\text {. }}$

In particular, if $L$ is a subgroup of $N$, then $Z(N)$ if a right-free module over $\mathbf{Z}(L)$, that is $\mathbf{Z}(N) \otimes_{\mathbf{Z}(L)}$ - is left exact (preserves injection). Analogously, if $i: L \rightarrow N$ is a right free inclusion of categories, then the left Kan extension $[4 ; 9]$ along $i$, thought of as a generalization of the tensor product above, is left exact. We use this left exactness in a "change of rings" type argument [2].

Definition. Given two categories, $L, N$, in Cat Ca $_{l}, L \rightarrow N$ an inclusion, we say $i$ is a right-free inclusion if for all $x, y \in I$,

$$
N(x, y) \leftleftarrows L(x, y) \amalg\left(\coprod_{z \in I} \mathbf{N}(z, y) \times L(x, z)\right)
$$

where $\mathbf{N}(x, y) \in N(x, y)$ is a fixed set for each $x, y \in I$, and $1 \notin \mathbf{N}(x, x)$ for all $x \in I$. The arrow maps $L(x, y)$ into $N(x, y)$ by inclusion and $\mathbf{N}(z, y) \times L(x, z)$ into $N(x, y)$ by composition. We will let $\mathbf{N}=\bigsqcup_{x, y \in I} \mathbf{N}(x, y)$ and refer to $\mathbf{N}$ as the set of generators over $L$.

The composition of two right-free inclusions is right-free: if the functors $i: L \rightarrow M$ and $j: M \rightarrow N$ are right-free inclusions, $\mathbf{M}$ and $\mathbf{N}$ the set of generators of $M$ over $L$, and $N$ over $M$, respectively, then using the definition above, we can write

$$
\begin{aligned}
N(x, y)=L(x, y) \amalg\left(\coprod_{z \in I}[\right. & (\mathbf{M}(z, y) \amalg \mathbf{N}(z, y) \\
& \left.\left.\left.\amalg\left(\coprod_{z^{\prime} \in I} \mathbf{N}\left(z^{\prime}, y\right) \times \mathbf{M}\left(z, z^{\prime}\right)\right)\right) \times L(x, z)\right]\right) .
\end{aligned}
$$

The set $\mathbf{M} \amalg \mathbf{N} \amalg\left(\amalg_{z \in I} \mathbf{N}(z, y) \times \mathbf{M}(x, z)\right)$ serves as a set of generators of $N$ over $L$. Every element of $M$ is in $N$ by inclusion and $(\mathbf{n}, \mathbf{m}) \in\left(\coprod_{z \in I} \mathbf{N}(z, y) \times\right.$ $\mathbf{M}(x, z))$ represents $\mathbf{n m} \in \mathbf{N}$, a generator in $N$ over $L$. We can now state the first theorem.

THeOREM 1. Given the following push-out diagram in $\mathrm{Cat}_{I}$,

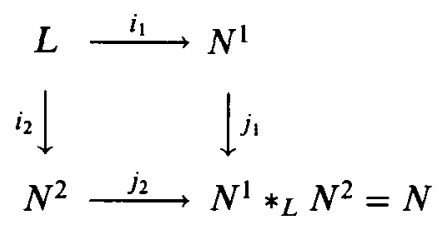

Diagram 2.1 
where $i_{1}, i_{2}$ are right-free inclusions, $\mathbf{N}^{i}$ the set of generators of $N^{j}, i=1,2$, over $L$, then every morphism of the push-out, $N^{1} *_{L} N^{2}$, can be represented uniquely by an (alternating) sequence $\left(\mathbf{n}_{\lambda}, \mathbf{n}_{\lambda-1}, \ldots, \mathbf{n}_{2}, \mathbf{n}_{1}, l\right), \lambda \geq 0$, where $l$ is a morphism in $L$, and $\mathbf{n}_{1}, \mathbf{n}_{2}, \ldots, \mathbf{n}_{\lambda}$ come alternately from $\mathbf{N}^{1}$ and $\mathbf{N}^{2}$, in such a way as to be composable in $N^{1} *_{L} N^{2}$.

Proof. Define the set $W_{x, y}=\left\{\left(\mathbf{n}_{\lambda}, \ldots, \mathbf{n}_{2}, \mathbf{n}_{1}, l\right)\right\}$, where $x \stackrel{l}{\rightarrow} x_{1} \stackrel{n_{1}}{\rightarrow} x_{2} \rightarrow$ $\cdots \rightarrow x_{\lambda} \stackrel{\mathrm{n}_{3}}{\rightarrow} y$. The graph, $W$, will consist of the objects, $I$, together with edges of the form $W_{x, y}$. The map $p: W \rightarrow N^{1} *_{L} N^{2}$, which is the identity on objects and maps $\left(\mathbf{n}_{\lambda}, \ldots, \mathbf{n}_{2}, \mathbf{n}_{1}, l\right) \mapsto \mathbf{n}_{\lambda} \circ \cdots \circ \mathbf{n}_{2} \circ \mathbf{n}_{1} \circ l$ is surjective since any element of $N^{1} *_{L} N^{2}$ can be factored in at least one way into an alternating string of coset representatives followed by an element in $L$.

We next define the category, $\mathfrak{W}$, with objects, $I$, and morphisms, $\mathfrak{W}(x, y)=$ $\amalg_{z \in I} \operatorname{Sets}\left(W_{x, z}, W_{y, z}\right)$, where composition of morphisms is just composition of set maps. We define the functor $f^{1}: N^{1} \rightarrow \mathfrak{W}^{\mathrm{op}}$, for $\alpha: x \rightarrow y$ in $N^{1}$, $\left(\mathbf{n}_{\lambda}, \ldots, \mathbf{n}_{2}, \mathbf{n}_{1}, l\right)$ in $W_{y, z}$, by

$$
\begin{aligned}
& f(\alpha)\left(\mathbf{n}_{\lambda}, \ldots, \mathbf{n}_{2}, \mathbf{n}_{1}, l\right) \\
& \quad= \begin{cases}\left(\mathbf{n}_{\lambda}, \ldots, \mathbf{n}_{2}, \mathbf{n}_{1}, \mathbf{n}, l^{\prime}\right) & \text { where } \mathbf{n}_{1} \in N^{2} \text { and } l \alpha=\mathbf{n} l^{\prime}, \\
\left(\mathbf{n}_{\lambda}, \ldots, \mathbf{n}_{2}, \mathbf{n}, l^{\prime}\right) & \text { where } \mathbf{n}_{1} \in N^{1} \text { and } \mathbf{n}_{1} l \alpha=\mathbf{n} l^{\prime} .\end{cases}
\end{aligned}
$$

We define $f^{2}: N^{2} \rightarrow \mathfrak{W}^{\text {op }}$ in the same way and observe that they agree on $L$, giving the following commutative diagram

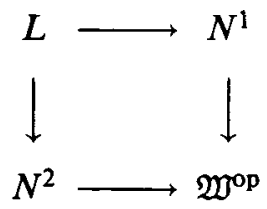

The universal property of push-outs yields a map $\Psi: N^{1} *_{L} N^{2} \rightarrow \mathfrak{W}^{\text {op }}$. We show that $p$ is injective by showing that $\Psi \circ p$ is injective. Consider $w=$ $\left(\mathbf{n}_{\lambda}, \ldots, \mathbf{n}_{2}, \mathbf{n}_{1}, l\right)$ in $W_{x, y}$. Then $\Psi \circ p(w)$ restricts to a map from $W_{y, y}$ to $W_{x, y}$. Since $\Psi$ is the unique map that makes the above diagram commute, then it must take the identity in $W_{y, y}$ to $\left(\mathbf{n}_{\lambda}, \ldots, \mathbf{n}_{2}, \mathbf{n}_{1}, l\right)$ in $W_{x, y}$. Thus different elements of $W$ yield different maps in $\mathfrak{W}^{\circ p}$, and $p$ must be injective, and hence $N^{1} *_{L} N^{2}$ is bijective correspondence with $W$.

COROLlary. The terminal legs, $j_{1}: N^{1} \rightarrow N^{1} *_{L} N^{2}$ and $j_{2}: N^{2} \rightarrow N^{1} *_{L} N^{2}$ are right-free inclusions with generators $\mathbf{G}^{1}=\left\{\left(\mathbf{n}_{\lambda}, \ldots, \mathbf{n}_{2}, \mathbf{n}_{1}, l\right) \in \mathbf{N} \mid \mathbf{n}_{1} \in \mathbf{N}^{2}\right\}$ and $\mathbf{G}^{2}=\left\{\left(\mathbf{n}_{\lambda}, \ldots, \mathbf{n}_{2}, \mathbf{n}_{1}, l\right) \in \mathbf{N} \mid \mathbf{n}_{1} \in \mathbf{N}^{1}\right\}$ and hence, so is the composition map $k=j_{1} i_{1}=j_{2} i_{2}: L \rightarrow N^{1} *_{L} N^{2}$ with generators $\mathbf{N}=\mathbf{G}^{1} \amalg \mathbf{G}^{2}$.

Proof. Since $i_{1}$ is a right-free inclusion, $n \in N^{1}$ can be written as $\mathbf{n} l$ in a unique fashion, with $\mathbf{n} \in \mathbf{N}^{1}, l \in L$. The map $j_{1}$ merely sends $n$ to $\mathbf{n} l$, 
its representation in $N^{1} *_{L} N^{2}$. So $j_{1}$ is clearly an inclusion. We have just shown an element in $N^{1} *_{L} N^{2}$ can be written as $n=\mathbf{n}_{\lambda} \circ \cdots \circ \mathbf{n}_{2} \circ \mathbf{n}_{1} \circ l$ in a unique way. If $\mathbf{n}_{1} \in \mathbf{N}^{2}$ then $n=\mathbf{n}_{\lambda} \circ \cdots \circ \mathbf{n}_{2} \circ n^{\prime}$ where $n^{\prime}=\mathbf{n}_{1} l \in N$ is clearly a unique representation of $n$ as the product of a generator and an element of $N^{1}$. Thus we see $\mathbf{G}^{1}=\left\{\left(\mathbf{n}_{\lambda}, \ldots, \mathbf{n}_{2}, \mathbf{n}_{1}, l\right) \in \mathbf{N} \mid \mathbf{n}_{1} \in \mathbf{N}^{2}\right\}$ gives us a set of generators of $N$ over $N^{1}$. We conclude $j_{1}$ is a right-free inclusion. We reason analogously to conclude $j_{2}$ is a right-free inclusion. It follows immediately that $k$, the composition of two right-free inclusions is a right-free inclusion with generators

$\mathbf{N}=\mathbf{N}^{1} \amalg \mathbf{G}^{1} \amalg\left[\coprod_{z \in I} \mathbf{G}^{1}(z, y) \times \mathbf{N}^{1}(x, z)\right]=\mathbf{N}^{2} \amalg \mathbf{G}^{2} \amalg\left[\coprod_{z \in I} \mathbf{G}^{2}(z, y) \times \mathbf{N}^{2}(x, z)\right]$.

We see that $\mathbf{G}^{1}=\mathbf{N}^{2} \amalg\left[\amalg_{z \in I} \mathbf{G}^{2}(z, y) \times \mathbf{N}^{2}(x, z)\right]$ and $\mathbf{G}^{2}=\mathbf{N}^{1} \amalg\left[\amalg_{z \in I} \mathbf{G}^{1}(z, y)\right.$ $\left.\times \mathbf{N}^{1}(x, z)\right]$. Then $\mathbf{N}=\mathbf{G}^{1} \amalg \mathbf{G}^{2}$.

\section{Preservation of homotopy type in the presence of right-free} inclusions: $B N^{1} \cup_{B L} B N^{2} \stackrel{\sim}{\rightarrow} B\left(N^{1} *_{L} N^{2}\right)$

Theorem 2. Given the following push-out diagram in Cat I

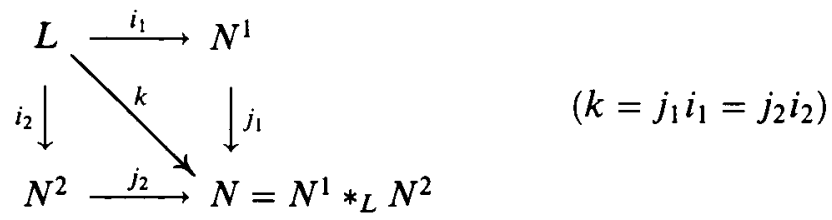

Diagram 3.1

with $i_{1}, i_{2}$ right-free inclusion of categories, then the map induced by applying the classifying functor, $\beta: B N^{1} \cup_{B L} B N^{2} \rightarrow B\left(N^{1} *_{L} N^{2}\right)$, is an equivalence.

Proof. The proof will proceed by showing

(I) $\Pi_{1}\left(B\left(N^{1} *_{L} N^{2}\right), x\right) \stackrel{\sim}{\rightarrow} \Pi_{1}\left(B N^{1} \cup_{B L} B N^{2}, x\right)$, and

(II) $\beta: B N^{1} \cup_{B L} B N^{2} \rightarrow B\left(N^{1} *_{L} N^{2}\right)$ induces an isomorphism in homology with all local coefficients.

Proof OF (I). Applying $\Gamma$, the groupoid reflection, to the above diagram yields $\Gamma N \stackrel{\sim}{\rightarrow} \Gamma N^{1}{ }^{*} \Gamma L N^{2}$ because $\Gamma$ is a left-adjoint and thus preserves pushouts. To get $\Pi_{1}$, we consider the vertex groups of these categories at $x$. 
Doing this yields the following diagram which in turn gives the desired result.

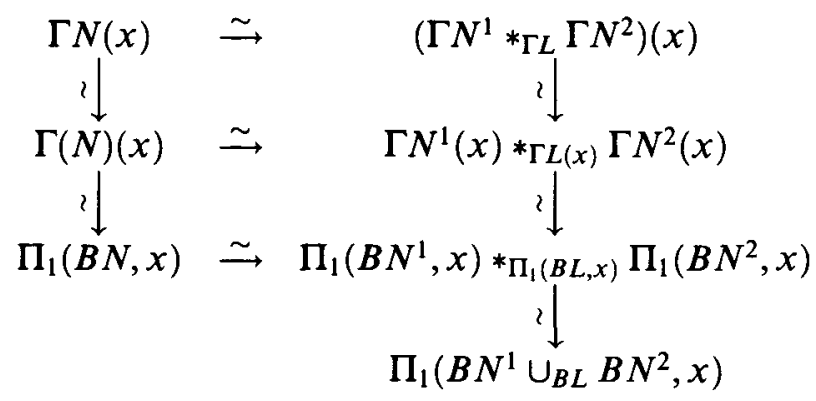

Since $i_{1}$ and $i_{2}$ are inclusions, we know that $\Gamma\left(i_{1}\right)$ and $\Gamma\left(i_{2}\right)$ are inclusions also, which implies that $\left(\Gamma N^{1} *_{\Gamma L} \Gamma N^{2}(x) \stackrel{\sim}{\rightarrow} \Gamma N^{1}(x){ }^{*} \Gamma L(x) \Gamma N^{2}(x)\right.$. For any category $N, \Gamma N(x) \stackrel{\sim}{\rightarrow} \Pi_{1}(B N, x)$ [11]. We use this to get both vertical isomorphisms for the bottom square. From the van Kampen theorem, we have $\Pi_{1}\left(B N^{1} \cup_{B L} B N^{2}, x\right) \stackrel{\sim}{\rightarrow} \Pi_{1}\left(B N^{1}, x\right) *_{\Pi_{1}(B L, x)} \Pi_{1}\left(B N^{2}, x\right)$, and thus we conclude that $\Pi_{1}\left(B\left(N^{1} *_{L} N^{2}\right), x\right) \stackrel{\sim}{\rightarrow} \Pi_{1}\left(B N^{1} \cup_{B L} B N^{2}, x\right)$.

Proof of (II). Again, considering Diagram 3.1, we let $T$ be a morphisminverting functor from $N$ to $\mathbf{A b}$ and 1 be the category with one object and one morphism. Then consider

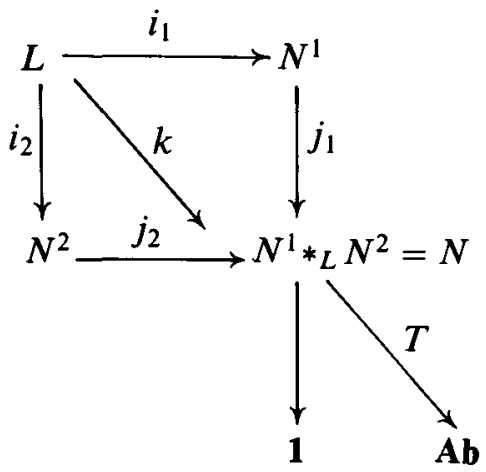

By first pulling $T$ back to each of the other categories, then taking the appropriate Kan extensions, we get the following commutative diagram in $\mathbf{A b}^{N}$.

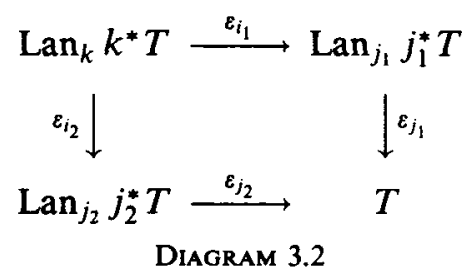


The maps in this diagram come from adjoint pairs of the form $\operatorname{Lan}_{j}, j^{*}$, where $j=i_{1}, i_{2}, j_{1}, j_{2}$ or $k$. For instance, $\operatorname{Lan}_{k} k^{*} T=\operatorname{Lan}_{j_{1}, i_{1}}\left(j_{1} i_{1}\right)^{*} T=$ $\operatorname{Lan}_{j_{1}}\left(\operatorname{Lan}_{i_{1}} i_{1}^{*}\right) j_{1}^{*} T \rightarrow \operatorname{Lan}_{j_{1}} j_{i}^{*} T$ by $\varepsilon_{i_{1}}$. This diagram commutes from straightforward application of properties of adjoints, Lan, etc. Further useful properties of this diagram are proved in the following lemmas.

LEMMA 1. The map $\left(\varepsilon_{i_{1}} \varepsilon_{i_{2}}\right): \operatorname{Lan}_{k} k^{*} T \rightarrow \operatorname{Lan}_{j_{1}} j_{1}^{*} T \oplus \operatorname{Lan}_{j_{2}} j_{2}^{*} T$ is an injection.

Proof. We observe that for $i: L \rightarrow M$ a right-free inclusion, $\mathbf{M}$, we may exploit the bijection $M(x, y) \simeq L(x, y) \amalg\left(\amalg_{z \in I} \mathbf{M}(z, y) \times L(x, z)\right)$ to write $\left(\operatorname{Lan}_{i} F\right)$ is a particularly simple way. We do this by writing $\left(\operatorname{Lan}_{i} F\right)(y)$ as a co-end (denoted by an integral sign), then using the fact that $N(x,-)$ is in Sets $^{\text {op }}$ [9, pages 236-239], we get

$$
\begin{aligned}
\left(\operatorname{Lan}_{i} F\right)(y) & =\int^{z} N(z, y) \times F(z) \\
& =\int^{z}\left[L(z, y) \amalg\left(\coprod_{z^{\prime} \in I} \mathbf{N}\left(z^{\prime}, y\right) \times L\left(z, z^{\prime}\right)\right)\right] \times F(z) \\
& =\int^{z}\left[L(z, y) \times F(z) \amalg\left[\coprod_{z^{\prime} \in I} \mathbf{N}\left(z^{\prime}, y\right) \times L\left(z, z^{\prime}\right)\right] \times F(z)\right],
\end{aligned}
$$

by the definition of right-free inclusion and the fact that colimits commute. The left Kan extension along an identity map leaves the functor unchanged so $\int^{z} L(z, y) \times F(z)$ is merely $F(y)$, and since $\mathbf{N}\left(z^{\prime}, y\right)$ contains no variable of integration, we pull it out from under the integral sign [9, page 210]. Thus

$$
\begin{aligned}
\left(\operatorname{Lan}_{i} F\right)(y) & =F(y) \amalg\left[\coprod_{z^{\prime} \in I} \mathbf{N}\left(z^{\prime}, y\right) \times \int^{z}\left[L\left(z, z^{\prime}\right) \times F(z)\right]\right] \\
& =F(y) \amalg\left[\coprod_{z^{\prime} \in I} \mathbf{N}\left(z^{\prime}, y\right) \times F\left(z^{\prime}\right)\right] .
\end{aligned}
$$

Thus we have the following expression

$$
\left(\operatorname{Lan}_{i} F\right)(y)=F(y) \amalg\left[\coprod_{z \in I} \mathbf{N}(z, y) \times F(z)\right] .
$$

Using this expression for $\left(\operatorname{Lan}_{i} F\right)(y)$, it is easy to see that $\operatorname{Lan}_{i}$ preserves injections whenever $i$ is a right-free inclusion. 
We can now rewrite Diagram 3.2, evaluated at $y$, in the following manner.

$$
\begin{aligned}
& T(y) \amalg\left(\coprod_{z \in I} \mathbf{N}(z, y) \times T(z)\right) \stackrel{\varepsilon_{i_{1}}}{\longrightarrow} T(y) \amalg\left(\coprod_{z \in I} \mathbf{G}^{1}(z, y) \times T(z)\right) \\
& T(y) \amalg\left(\coprod_{i_{i_{1}}} \downarrow\right. \\
& \left.\mathbf{G}^{2}(z, y) \times T(z)\right) \longrightarrow T(y)
\end{aligned}
$$

This diagram is in the category of abelian groups. The maps are clear for elements $T(y)$. By the corollary to Theorem 1 we have $\mathbf{N}=\mathbf{G}^{1} \amalg \mathbf{G}^{2}$. If $(\mathbf{n}, t)$ is such that $\mathbf{n} \in \mathbf{G}^{1}$, then $\mathbf{n}=\mathbf{g}^{\prime} \mathbf{n}^{\prime}$, where $\mathbf{g}^{\prime} \in \mathbf{G}^{2}$ or $\mathbf{g}^{\prime}=1$ and $\mathbf{n}^{\prime} \in \mathbf{N}^{1}$. In this case $(\mathbf{n}, t)$ is mapped as in the diagram below.

$$
\begin{gathered}
(\mathbf{n}, t) \quad \stackrel{\varepsilon_{i_{1}}}{\longrightarrow} \quad(\mathbf{n}, t) \\
\varepsilon_{i_{2}} \downarrow \\
\left(\mathbf{g}^{\prime}, T\left(\mathbf{n}^{\prime}\right)(t)\right) \stackrel{\downarrow}{\longrightarrow}\left(T\left(\mathbf{g}^{\prime}\right) T\left(\mathbf{n}^{\prime}\right)(t)\right)=(T(\mathbf{n})(t))
\end{gathered}
$$

A similar statement holds in the case that $\mathbf{n} \in \mathbf{G}^{2}$.

Let $(\mathbf{n}, t) \in \operatorname{ker} \varepsilon_{i_{1}} \cap \operatorname{ker} \varepsilon_{i_{2}}$. Suppose that $\mathbf{n} \in \mathbf{G}^{1}$, then

$$
\left(\mathbf{g}^{\prime}, T\left(\mathbf{n}^{\prime}\right)(t) \stackrel{\varepsilon_{i_{2}}}{\longrightarrow}(\mathbf{n}, t) \stackrel{\varepsilon_{i_{1}}}{\longrightarrow}(\mathbf{n}, t)=(\mathbf{n}, 0)\right.
$$

which implies $t=0$. The argument is the same if $\mathbf{n} \in \mathbf{G}^{2}$, and thus $\operatorname{ker}\left(\varepsilon_{i_{1}} \varepsilon_{i_{2}}\right)$ $=\{0\}$, and $\left(\varepsilon_{i_{1}} \varepsilon_{i_{2}}\right)$ is an injection.

LeMMA 2. Diagram 3.2 is a pushout.

Proof. We consider the following diagram in Sets $^{\mathbf{N}}$.

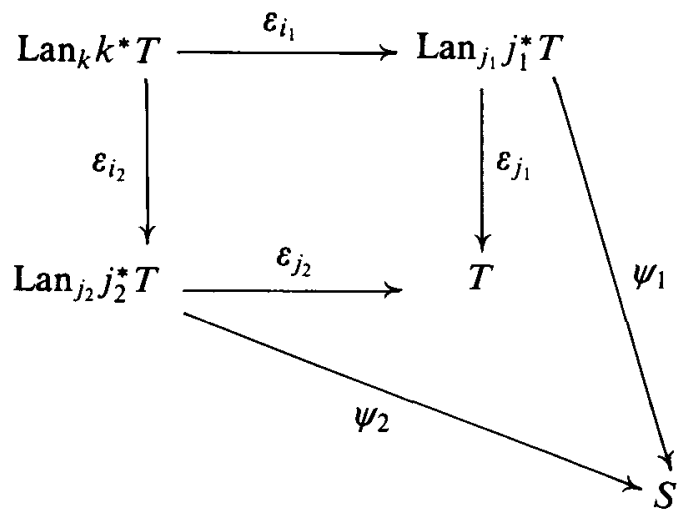

Given $\psi_{2} \varepsilon_{i_{2}}=\psi_{1} \varepsilon_{i_{1}}$, we obtain $\theta: T \rightarrow S$ from the following commutative diagram. 


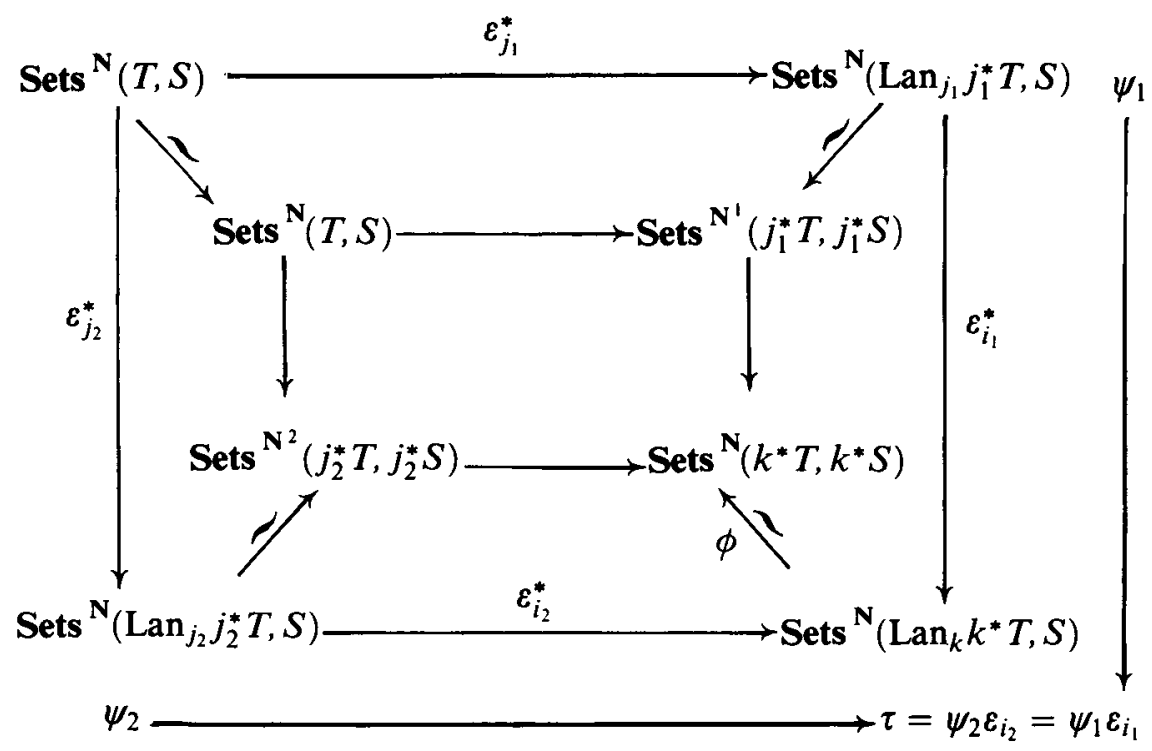

We take the adjunctions of $\psi_{1}, \psi_{2}$, and $\tau=\psi_{2} \varepsilon_{i_{2}}=\psi_{1} \varepsilon_{i_{1}}$, thereby moving from the outer square to the inner square. These adjunctions agree on objects, so we can define $\theta=\phi \tau$ and show it will satisfy the necessary commutativity conditions for naturality.

From the word theorem, any $w \in N=N^{1} *_{L} N^{2}$ can be written uniquely as $w=\mathbf{n}_{\lambda} \mathbf{n}_{\lambda-1} \cdots \mathbf{n}_{2} \mathbf{n}_{1} l$ where the $\mathbf{n}_{\lambda}$ come alternately from $\mathbf{N}^{1}$ and $\mathbf{N}^{2}, l \in L$. If $x \rightarrow y$, for $x, y \in N$, then the following diagram

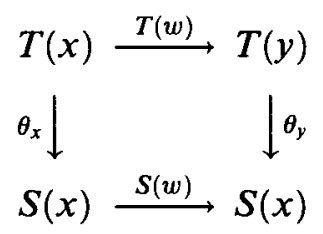

Diagram 3.4

can be expanded to the following form

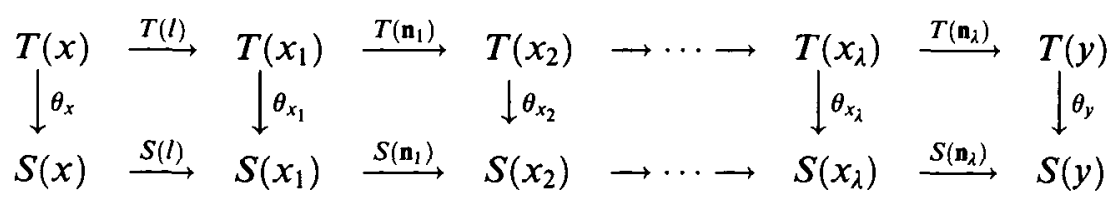

where $x \stackrel{\iota}{\rightarrow} x_{1} \stackrel{\mathbf{n}_{1}}{\rightarrow} x_{2} \rightarrow \cdots \rightarrow x_{\lambda} \stackrel{\mathbf{n}_{2}}{\rightarrow} y$. Each square in this ladder lies entirely in $L, N^{1}$, or $N^{2}$, and commutes because $\theta$ is a natural transformation in the appropriate category. 
The commutativity of each square gives the commutativity of Diagram 3.4. Thus, $\theta$ is a natural transformation from $T$ to $S$. The uniqueness of $\theta$ follows from the commutativity requirements it must satisfy and the universal property of the left Kan extension.

We now complete the proof of (II). Since Diagram 3.2 is in $\mathbf{A b}^{\mathbf{N}}$, an abelian category, Lemmas 1 and 2 give the following exact sequence.

$$
0 \rightarrow \operatorname{Lan}_{k} k^{*} T \rightarrow \operatorname{Lan}_{j_{1}} j_{1}^{*} T \oplus \operatorname{Lan}_{j_{2}} j_{2}^{*} T \rightarrow T \rightarrow 0
$$

This, in turn, yields the long exact sequence

$$
\begin{aligned}
\cdots \rightarrow H_{n}\left(N, \operatorname{Lan}_{k} k^{*} T\right) \rightarrow H_{n}\left(N, \operatorname{Lan}_{j_{1}} j_{1}^{*} T\right) \\
\qquad H_{n}\left(N, \operatorname{Lan}_{j_{2}} j_{2}^{*} T\right) \rightarrow H_{n}(N, T) \rightarrow \cdots
\end{aligned}
$$

from the commutativity of $H_{n}$ with $\oplus$.

Since for any right-free inclusion, $i: M \rightarrow N$, $\operatorname{Lan}_{1}$ preserves injections, we have $H_{n}\left(N, \operatorname{Lan}_{i} i^{*} T\right) \simeq H_{n}\left(M, i^{*} T\right)$. Thus we may rewrite the long exact sequence to yield the top row of the following diagram.

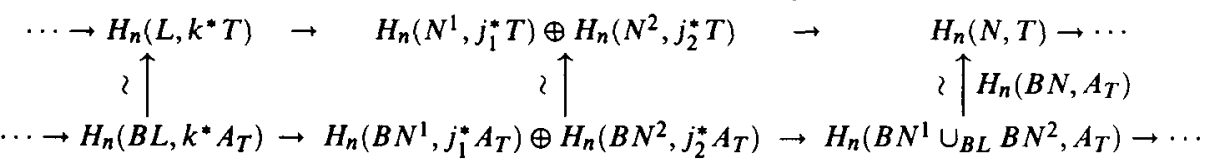

where $A_{T}$ is the local coefficient system associated with $T$. Using the 5Lemma we get $H_{n}\left(B N^{1} \cup_{B L} B N^{2}, A_{T}\right) \stackrel{\sim}{\rightarrow} H_{n}\left(B\left(N^{1} *_{L} N^{2}\right), A_{T}\right)$.

\section{Construction of a mapping cylinder}

Let $L, N$ be two categories in Cat $I$ and $f: L \rightarrow N$ be a functor which is the identity on objects. The following theorem allows us to construct a mapping cylinder, $\operatorname{Cyl}(f)$, for the functor $f$.

THEOREM 3. There exists a factorization of $f$

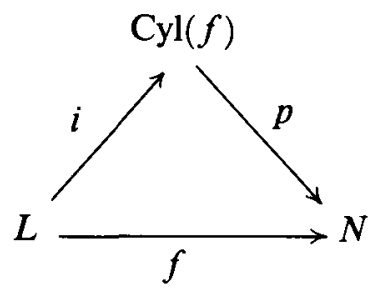

with the following properties:

(i) $p i=f$;

(ii) $i$ is a right-free inclusion;

(iii) $p$ is a homotopy equivalence. 
Proof. We describe the mapping cylinder in terms of generators and relations, that is,

$$
\begin{array}{r}
\operatorname{Cyl}(f)=\left\langle L, M, t_{x}\right| t_{y} \alpha=\alpha t_{x}, t_{y} \beta=\beta t_{x}, t_{y} \alpha=t_{y} f(\alpha), \\
\text { and } \left.t_{x}^{2}=t_{x}, \text { for } \alpha, \beta: x \rightarrow y, \alpha \in L, \beta \in N, x, y \in I\right\rangle .
\end{array}
$$

Consider the following diagram in Cat $_{I}$.

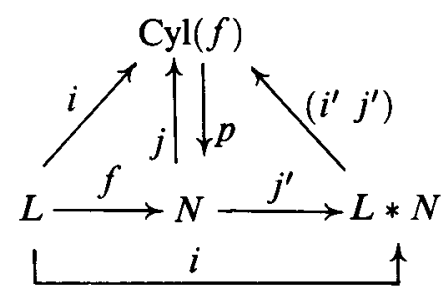

The functors $i, j, i^{\prime}, j^{\prime}$ are the obvious inclusions, and $p$ is defined as the identity on objects and on generators of $\operatorname{Cyl}(f)$ by

$$
\begin{aligned}
& p(\alpha)=f(\alpha), \quad \alpha \in L, \\
& p(\beta)=\beta, \quad \beta \in N, \\
& p\left(t_{x}\right)=1_{x} .
\end{aligned}
$$

Property (i) is satisfied by definition of $p$. To show property (ii), that the inclusion $i: L \rightarrow \operatorname{Cyl}(f)$ is right-free, we note that $i^{\prime}$ is right-free inclusion and show that $\left(i^{\prime} j^{\prime}\right)$ is also. Then $i=\left(i^{\prime} j^{\prime}\right) \circ i^{\prime}$ will be a right-free inclusion, since composition preserves this property. We now show that $\left(i^{\prime} j^{\prime}\right): L * N \rightarrow$ $\operatorname{Cyl}(f)$ is a right free inclusion with generators $\operatorname{Cyl}(f)=\left\{t_{x} \mid x \in I\right\}$. It is clear that $\left(i^{\prime} j^{\prime}\right)$ is an inclusion and we need only show that any element $\gamma \in \operatorname{Cyl}(f)$ can be written in a unique way as $w$ or $t_{y} w$ where $w \in L * N$. Note that the defining relations of $\operatorname{Cyl}(f)$ and the definition of $p$ imply that for $w \in L * N$, $w t_{x}=t_{y} w=t_{y} p(w)$, suppressing the notation for the inclusion, $j$. Any element, $\gamma$, in $\operatorname{Cyl}(f) \backslash L * N$ can be written as the product of generators, that is, $\gamma=w_{1} t w_{2} t \cdots t w_{k}$ where $w_{i} \in L * N$. Then $\gamma=t w_{1} w_{2} \cdots w_{k}=$ $t p\left(w_{1} w_{2} \cdots w_{k}\right)$. Observe that $p(\gamma)=p\left(w_{1}\right) p\left(w_{2}\right) \cdots p\left(w_{k}\right)=p\left(w_{1} w_{2} \cdots w_{k}\right)$, so we can write $\gamma=t p(\gamma)$. The uniqueness follows immediately from the fact that $\gamma_{1}=\gamma_{2}$ implies that $p\left(\gamma_{1}\right)=p\left(\gamma_{2}\right)$.

We know that $p_{j}=1_{N}$, so to show that $p$ is a homotopy equivalence, it is sufficient to show there is a natural transformation $j p \rightarrow 1_{\mathrm{Cyl}(f)}$ since such a transformation becomes a homotopy when we apply the classifying functor. We define $T: j p \rightarrow 1_{\mathrm{Cyl}(f)}$ by $T_{x}=t_{x}$ and observe that for $\gamma: x \rightarrow y$ in $\operatorname{Cyl}(f)$ 
the following diagram commutes, showing that $T$ satisfies the conditions for naturality.

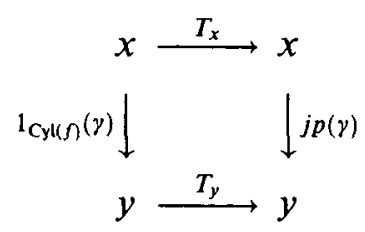

Thus, $p j=1_{N}$ and $1_{C y l(f)}=j p$, and we conclude that $p$ is homotopy equivalence.

\section{Embedding a category into semi-groupoid of the same homotopy type}

To prove the last theorem, we need the following result from graph theory: every connected category, $L$, has a contractible subcategory, $L_{0}$, with at most one morphism between any two objects (that is, $L_{0}$ is a preorder), and $\mathrm{Ob} L=$ $\mathrm{Ob} L_{0}$.

We are now ready to prove our main result.

THEOREM 4. Given a connected category $L$, we can embed it by a rightfree inclusion in a semi-groupoid, $N$, where the inclusion induces a homotopy equivalence.

Proof. Let $L_{0}$ be a contractible subcategory of $L$, with $\mathrm{Ob} L_{0}=\mathrm{Ob} L$ and $I\left(L_{0}\right)$ the indiscrete category containing $L_{0}$. Using the mapping cylinders of $i_{L}: L_{0} \rightarrow L$ and $i_{I}: L_{0} \rightarrow I\left(L_{0}\right)$, respectively, we take the push-out indicated in the diagram below.

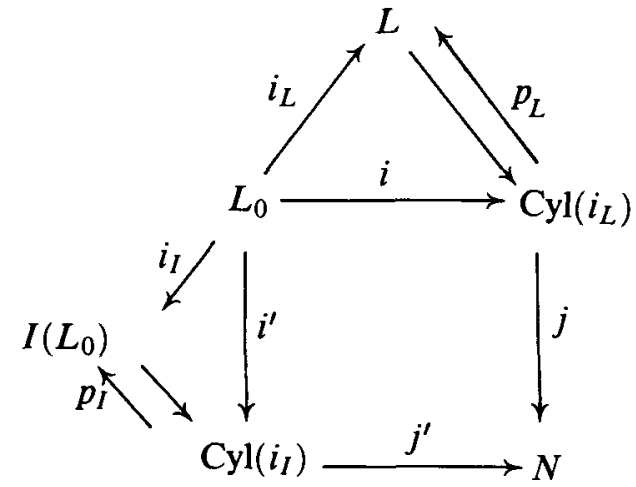


The maps $i$ and $i^{\prime}$ are the right-free inclusions of $L_{0}$ into the mapping cylinders $\operatorname{Cyl}\left(i_{L}\right)$ and $\operatorname{Cyl}\left(i_{I}\right)$, respectively. Thus the terminal legs, $j$ and $j^{\prime}$, of this push-out are right-free inclusions, by the corollary to Theorem 1 . The map $I\left(L_{0}\right) \rightarrow \operatorname{Cyl}\left(i_{I}\right)$ is a right-free inclusion by Theorem 3 , therefore $I\left(L_{0}\right)$ is contained in $N$ as a subcategory, making $N$ a semi-groupoid. Then by Theorem 2, we have $B N \simeq B\left(\operatorname{Cyl}\left(i_{L}\right)\right) \cup_{B L_{0}} B\left(\operatorname{Cyl}\left(i_{I}\right)\right) \simeq B L \cup_{B L_{0}} B L_{0} \simeq B L$.

Since any semi-groupoid is the product of a contractible category and a monoid, the homotopy type of a monoid at any vertex of a semi-groupoid is the same as that of the entire semi-groupoid. Thus, for any connected category in Cat I $_{I}$, we can find a monoid with the same homotopy type.

The mapping cylinder is constructed so that, if we start with a category, $L$, presented in terms of generators and relations, then the mapping cylinder constructed from $L$ will be in terms of generators and relations. This implies the same is true of the semi-groupoid constructed in Theorem 4. If we denote by $N\left(x_{0}, x_{0}\right)$ the monoid at a fixed vertex, $x_{0}$, of $N$, then $\phi: N \rightarrow I\left(L_{0}\right) \times$ $N\left(x_{0}, x_{0}\right)$ takes $n: x \rightarrow y$ to $\left(l, l^{-1} n\right):\left(x, x_{0}\right) \rightarrow\left(y, x_{0}\right)$, where $l \in I\left(L_{0}\right), n \in$ $N$, and $l: x \mapsto y$. By composing with the canonical projection onto $N\left(x_{0}, x_{0}\right)$, we have a surjection of $N$ onto $N\left(x_{0}, x_{0}\right)$. Using this surjection, we can easily show that the monoid at any fixed vertex is presented by the generators and relations of $N$ pulled back to that fixed vertex by the isomorphisms provided by $I\left(L_{0}\right)$.

The cardinality of new morphisms introduced is that of $I$, the cardinality of new relations is that of the morphisms of $L$. If $L$ is finitely or countably presented then the associated monoid can be presented likewise. This construction can be carried out explicitly once the selection of a spanning tree for $L$ has been made.

\section{References}

[1] G. Baumslag, E. Dyer and A. Heller, "The topology of discrete groups," J. Pure Appl. Algebra 16 (1980), 1-47.

[2] H. Cartan and S. Eilenberg, Homological algebra (Princeton Univ. Press, Princeton, N.J., $1956,116-119$ and $149-150)$.

[3] P. J. Hilton and U. Stammbach, A course in homological algebra (Springer-Verlag, New York, Heidelberg, Berlin, 1971).

[4] D. M. Kan, “Adjoint functors,” Trans. Amer. Math. Soc. 87 (1958), 294-329.

[5] D. M. Kan and W. P. Thurston, "Every connected space has the homology of a $K(\pi, 1)$," Topology 15 (1976), 235-258.

[6] A. G. Kurosh, The theory of groups, transl. by K. A. Hirsch (Chelsea, 1956).

[7] D. M. Latch, "The uniqueness of homology for the category of small categories," J. Pure Appl. Algebra 9 (1977), 221-237. 
[8] D. M. Latch and R. Fritsch, "Homotopy inverses for nerve," Bull. Amer. Math. Soc. (N.S.) 1 (1979), 258-262.

[9] S. Mac Lane, Categories for the working mathematician (Springer-Verlag, New York, 1971).

[10] D. McDuff, "On the classifying spaces of discrete monoids," Topology 18 (1979), 313-319.

[11] D. G. Quillen, "Higher algebraic $K$-theory I," Algebraic k-theory I, pp. 85-147 (Lecture Notes in Mathematics, 341, Springer, New York, Heidelberg, Berlin, 1973).

[12] G. G. Segal, "Classifying spaces and spectral sequences," Publications in Mathematics 34, pp. 105-112 (Institute Haute Etudes Science, 1968).

[13] J. H. C. Whitehead, "On the asphericity of regions on a 3-sphere," Fund Math. 32 (1939), 149-166.

[14] J. H. C. Whitehead, "Combinatorial homotopy. I," Bull. Amer. Math. Soc. 55 (1949), 213245.

\author{
William Paterson College \\ Department of Mathematics \\ Wayne, New Jersey 07470 \\ U.S.A.
}

